\title{
CYCLE POLYNOMIALS
}

\author{
F. K. HWANG
}

\begin{abstract}
Let $G$ be a graph consisting of $\boldsymbol{m}$ vertex-disjoint cycles with possibly different numbers of vertices on each cycle. We want to count the number of ways of selecting $k$ vertices in $G$ such that there are exactly $l$ edges spanned by these $k$ vertices. For $m=1$, the problem is equivalent to the Whitworth bracelet problem with two colors and a closed-form solution is known. In this paper we show that the solution for the many-cycle case can be written as a sum of the solutions for single-cycle cases.
\end{abstract}

1. Introduction. Let $G$ be a graph consisting of $m$ vertex-disjoint cycles $C_{1}, \ldots, C_{m}$ where $C_{i}$ has $n_{i}$ vertices. We want to count the number of ways of selecting $k$ vertices in $G$ such that the subgraph induced by the $k$ selected vertices contains exactly $l$ edges. The problem originated from a study of blocking probabilities for certain types of switching networks [3], [5], [6] (Theorem 3 of this paper was used in [3]), but it also has connections with some well-studied mathematical problems.

For a single cycle, i.e., $m=1$, the problem is equivalent to the Whitworth bracelet $^{1}$ problem with two colors [1], [2], [4], [7] and a closed-form solution is known. For the general case, define $S=\left\{n_{1}, \ldots, n_{m}\right\}$. Let $g_{s, k}(t)$ be the generating function with given $S$ and $k$ such that the coefficient of the term $t^{l}$ is the number of ways of obtaining $l$ edges. We show that $g_{s, k}(l)$ can be represented as a sum of generating functions for the single cycle cases.

2. The single-cycle case. Let $G$ be a cycle with $n$ vertices. Suppose $k$ vertices in $G$ are selected. If $k=n$, then there is only one way of selecting vertices and it yields $n$ edges. If $k=0$, then again there is only one way of selecting vertices, and it has no edge. If $n>k>0$, then a selection partitions the vertices in $G$ into two types, those selected and those not selected. Let us call the two types the black beads and the white beads. Then, if the $k$ black beads contain $l$ edges between them, there must be $k-l$ runs of black beads and there must also be $k-l$ runs of white beads. Therefore, the problem becomes one of finding the number of ways to arrange $k$ black beads and $n-k$ white beads on a cycle (with labeled positions) into $2(k-l)$ runs, which is also known as the Whitworth bracelet problem. The

Received by the editors July 12, 1979 and, in revised form, May 27, 1980.

1980 Mathematics Subject Classification. Primary 05A15.

Key words and phrases. Whitworth runs, Jablonski runs, Whitworth bracelet problem.

${ }^{1}$ To be stated below.

(C) 1981 American Mathematical Society 0002-9939/81/0000-0449/\$02.25 
number of ways of having a total of $2(k-l)$ runs is (see [2])

$$
\frac{n\left(\begin{array}{c}
k-1 \\
k-l-1
\end{array}\right)\left(\begin{array}{l}
n-k-1 \\
k-l-1
\end{array}\right)}{k-l}
$$

This can be derived by considering the cycle as obtained from bending a line which contains either $2(k-l)$ or $2(k-l)+1$ runs. Summarizing, we have

THEOREM 1.

$$
g_{\{n\}, k}(t)= \begin{cases}t^{n}, & k=n>0 \\
1, & k=0 \\
n \sum_{l=0}^{k-1} \frac{\left(\begin{array}{c}
k-1 \\
k-l-1
\end{array}\right)\left(\begin{array}{l}
n-k-1 \\
k-l-1
\end{array}\right)}{k-l} t^{l}, & n>k>0 \\
0, & \text { otherwise. }\end{cases}
$$

\section{The two-cycle case. Define}

$$
\Delta_{\left\{n_{1}, n_{2}\right\}, k}(t)=g_{\left\{n_{1}, n_{2}\right\}, k}(t)-g_{\left\{n_{1}+n_{2}\right\}, k}(t)
$$

We first prove a recursive equation.

LEMMA 1.

$$
\Delta_{\left\{n_{1}, n_{2}\right\}, k}(t)=(t-1) \Delta_{\left\{n_{1}-1, n_{2}-1\right\}, k-1}(t) \text { for } n_{1}, n_{2}>1 \text {. }
$$

Proof. We will regard the cycle $C_{n_{1}+n_{2}}$ as obtained from the union of two cycles $C_{n_{1}}$ and $C_{n_{2}}$ by deleting one edge $[A, B]$ in $C_{n_{1}}$, one edge $[C, D]$ in $C_{n_{2}}$ and adding the edges $[A, C]$ and $[B, D]$ (see Figure 1 ).

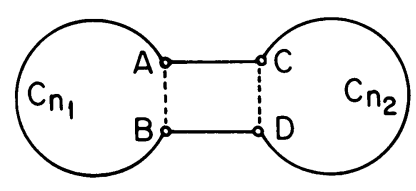

FIGURE 1. $C_{n_{1}+n_{2}}$ as obtained from $C_{n_{1}} \cup C_{n_{2}}$

Consider a particular selection of $k$ vertices. If the four vertices $A, B, C$ and $D$ are either all selected or all not selected, then the contribution to $\Delta_{\left\{n_{1}, n_{2}\right\}, k}(t)$ is clearly zero. Furthermore, if any three of the four vertices are selected, then the contribution to $\Delta_{\left\{n_{1}, n_{2}\right\}, k}(t)$ is again zero since the three selected vertices will yield two edges, one appearing in $C_{n_{1}} \cup C_{n_{2}}$ and the other in $C_{n_{1}+n_{2}}$, and thus cancelling each other. Therefore, the contribution to $\Delta_{\left\{n_{1}, n_{2}\right\}, k}(t)$ is nonzero only if exactly two vertices from $A, B, C$ and $D$ are selected. In these cases, the two graphs $C_{n_{1}} \cup C_{n_{2}}$ and $C_{n_{1}+n_{2}}$ will differ by one edge.

Let $g_{s, k}\left(t \mid x_{1}, \ldots, x_{p}, \bar{y}_{1}, \ldots, \bar{y}_{q}\right)$ denote the conditional generating function given vertices $x_{1}, \ldots, x_{p}$ are selected and vertices $y_{1}, \ldots, y_{q}$ are not. If $A$ and $B$ are selected but not $C$ and $D$, then

$$
\Delta_{\left\{n_{1}, n_{2}\right\}, k}(t \mid A B \bar{C} \bar{D})=(t-1) g_{\left\{n_{1}+n_{2}\right\}, k}(t \mid A B \bar{C} \bar{D})
$$


Similarly, if $C$ and $D$ are selected but not $A$ and $B$, then

$$
\Delta_{\left\{n_{1}, n_{2}\right\}, k}(t \mid C D \bar{A} \bar{B})=(t-1) g_{\left\{n_{1}+n_{2}\right\}, k}(t \mid C D \bar{A} \bar{B}) .
$$

On the other hand, in the case that either the pair $(A, C)$ or the pair $(B, D)$ is selected, but not both, then

$$
\Delta_{\left\{n_{1}, n_{2}\right\}, k}(t \mid A C \bar{B} \bar{D})=-(t-1) g_{\left\{n_{1}, n_{2}\right\}, k}(t \mid A C \bar{B} \bar{D})
$$

or

$$
\Delta_{\left\{n_{1}, n_{2}\right\}, k}(t \mid B D \bar{A} \bar{C})=-(t-1) g_{\left\{n_{1}, n_{2}\right\}, k}(t \mid B D \bar{A} \bar{C}) .
$$

Let $C_{n_{1}-1}, C_{n_{2}-1}$, and $C_{n_{1}+n_{2}-2}$ be the cycles obtained from $C_{n_{1}}, C_{n_{2}}$, and $C_{n_{1}+n_{2}}$ by merging the pairs of vertices $(A, B),(C, D),(A, C)$ and $(B, D)$ into single vertices $W, X, Y$ and $Z$ respectively. Then we can rewrite the conditional generating function in terms of the new cycles, for example,

$$
\begin{aligned}
g_{\left\{n_{1}+n_{2}\right\}, k}(t \mid A B \bar{C} \bar{D}) & =g_{\left\{n_{1}-1, n_{2}-1\right\}, k-1}(t \mid W \bar{X}), \\
g_{\left\{n_{1}, n_{2}\right\}, k}(t \mid A C \bar{B} \bar{D}) & =g_{\left\{n_{1}+n_{2}-2\right\}, k-1}(t \mid Y \bar{Z}) .
\end{aligned}
$$

But

$$
\begin{aligned}
& g_{\left\{n_{1}-1, n_{2}-1\right\}, k-1}(t \mid W \bar{X})+g_{\left\{n_{1}-1, n_{2}-1\right\}, k-1}(t \mid X \bar{W}) \\
& \quad=g_{\left\{n_{1}-1, n_{2}-1\right\}, k-1}(t)-g_{\left\{n_{1}-1, n_{2}-1\right\}, k-1}(t \mid W X)-g_{\left\{n_{1}-1, n_{2}-1\right\}, k-1}(t \mid \bar{W} \bar{X})
\end{aligned}
$$

and

$$
\begin{aligned}
& g_{\left\{n_{1}+n_{2}-2\right\}, k-1}(t \mid Y \bar{Z})+g_{\left\{n_{1}+n_{2}-2\right\}, k-1}(t \mid Z \bar{Y}) \\
& \quad=g_{\left\{n_{1}+n_{2}-2\right\}, k-1}(t)-g_{\left\{n_{1}+n_{2}-2\right\}, k-1}(t \mid Y Z)-g_{\left\{n_{1}+n_{2}-2\right\}, k-1}(t \mid \bar{Y} \bar{Z}) .
\end{aligned}
$$

Furthermore,

$$
g_{\left\{n_{1}-1, n_{2}-1\right\}, k-1}(t \mid X W)=g_{\left\{n_{1}+n_{2}-2\right\}, k-1}(t \mid Y Z)
$$

and

$$
g_{\left\{n_{1}-1, n_{2}-1\right\}, k-1}(t \mid \bar{X} \bar{W})=g_{\left\{n_{1}+n_{2}-2\right\}, k-1}(t \mid \bar{Y} \bar{Z}) .
$$

Summing up these equations, we obtain Lemma 1. Next we show

LeMma 2.

$$
g_{\left\{n_{1}, 1\right\}, k}(t)-g_{\left\{n_{1}+1\right\}, k}(t)=(t-1) g_{\left\{n_{1}-1\right\}, k-1}(t) \text { for } n_{1}>1 .
$$

Proof. Again we will regard the cycle $C_{n_{1}+1}$ as obtained from the union of two cycles $C_{n_{1}}$ and $C_{1}$ by deleting one edge $[A, B]$ in $C_{n_{1}}$ and the edge $[C, C]$ in $C_{1}$ (see Figure 2).

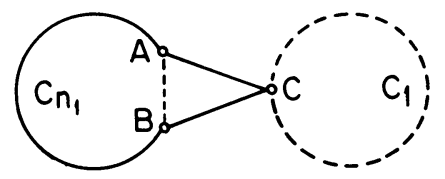

FIGURE 2. $C_{n_{1}+1}$ as obtained from $C_{n_{1}} \cup C_{1}$ 
Then

$$
\begin{aligned}
\Delta_{\left\{n_{1}, 1\right\}, k}(t) & =(t-1)\left[g_{\left\{n_{1}+1\right\}, k}(t \mid A B \bar{C})+g_{\left\{n_{1}+1\right\}, k}(t \mid C \bar{A} \bar{B})\right] \\
& =(t-1) g_{\left\{n_{1}-1\right\}, k-1}(t) .
\end{aligned}
$$

Finally, it is straightforward to verify

LeMma 3.

$$
\Delta_{\{1,1\}, k}(t)= \begin{cases}2(t-1), & k=1 \\ 0, & \text { otherwise }\end{cases}
$$

Theorem 2 in the following is now a direct consequence of Lemmas 1, 2 and 3. TheOREM 2.

$$
\begin{aligned}
g_{\left\{n_{1}, n_{2}\right\}, k}(t)= & g_{\left\{n_{1}+n_{2}\right\}, k}(t)+(t-1)^{n_{1}} g_{\left\{n_{2}-n_{1}\right\}, k-n_{1}}(t) \\
& +(t-1)^{n_{2}} g_{\left\{n_{1}-n_{2}\right\}, k-n_{2}}(t) .
\end{aligned}
$$

4. The general case. Let $S$ denote the set $\left\{n_{1}, \ldots, n_{m}\right\}$ and $S^{\prime}$ the subset $\left\{n_{1}, \ldots, n_{m-1}\right\}$. Let $s\left(s^{\prime}\right)$ denote a nonempty proper subset of $S\left(S^{\prime}\right)$ and $n(s)$ $\left(n\left(s^{\prime}\right)\right)$ the sum of $n_{i}$ in $s\left(s^{\prime}\right)$.

TheOREM 3.

$$
g_{S, k}(t)=g_{\{n(S)\}, k}(t)+\sum_{s}(t-1)^{n(s)} g_{\{n(S)-2 n(s)\}, k-n(s)}(t) \text { for } m>2
$$

Proof. We first write $g_{S, k}(t)$ as a convolution of $g_{S^{\prime}, i}(t)$ and $g_{\left\{n_{m}\right\}, k-i}(t)$. Then we use induction to expand $g_{S^{\prime}, i}(t)$. By convoluting $g_{\left\{n_{m\}}\right\}, k-i}(t)$ with each term in the expansion we obtain a sum of $g$ functions defined on two cycles. Then we use Theorem 2 to transform these $g$ functions on two cycles into $g$ functions of one cycle. By carefully counting terms, we obtain the right-hand side of Theorem 3 . The details of the proof are given in the Appendix.

5. Some concluding remarks. In this paper we essentially solve the problem of counting Whitworth runs of two colors in many cycles. Some possible topics for future research are to generalize the results to runs of many colors or to graphs other than cycles. Another possibility is to study the same problem for cycles whose vertices are not labeled. For the single-cycle case, the runs are known as Jablonski runs [1] and solutions for small numbers of beads and up to four colors have been tabulated in [1]. But the problem is open for two or more cycles.

Appendix.

Proof of Theorem 3. The case $m=2$ was proved in Theorem 2. We prove the general case by induction on $m$ and by using Theorem 2 . 


$$
\begin{aligned}
& g_{S, k}(t)=\sum_{i=0}^{k} g_{S^{\prime}, i}(t) g_{\left\{n_{m}\right\}, k-i}(t) \\
& =\sum_{i=0}^{k}\left[g_{\left\{n\left(S^{\prime}\right)\right\}, i}(t)+\sum_{s^{\prime}}(t-1)^{n\left(s^{\prime}\right)} g_{\left\{n\left(S^{\prime}\right)-2 n\left(s^{\prime}\right)\right\}, i-n\left(s^{\prime}\right)}(t)\right] g_{\left\{n_{m}\right\}, k-i}(t) \\
& =g_{\left\{n\left(S^{\prime}\right), n_{m}\right\}, k}(t)+\sum_{s^{\prime}}(t-1)^{n\left(s^{\prime}\right)} g_{\left\{n\left(S^{\prime}\right)-2 n\left(s^{\prime}\right), n_{m}\right\}, k-n\left(s^{\prime}\right)}(t) \\
& =g_{\{n(S)\}, k}(t)+(t-1)^{n\left(S^{\prime}\right)} g_{\left\{n_{m}-n\left(S^{\prime}\right)\right\}, k-n\left(S^{\prime}\right)}(t) \\
& +(t-1)^{n_{m}} g_{\left\{n\left(S^{\prime}\right)-n_{m}\right\}, k-n_{m}}(t)+\sum_{s^{\prime}}(t-1)^{n\left(s^{\prime}\right)} \\
& \cdot\left[g_{\left\{n(S)-2 n\left(s^{\prime}\right)\right\}, k-n\left(s^{\prime}\right)}(t)\right. \\
& +(t-1)^{n\left(S^{\prime}\right)-2 n\left(s^{\prime}\right)} g_{\left\{n_{m}-n\left(S^{\prime}\right)+2 n\left(s^{\prime}\right)\right\}, k-n\left(S^{\prime}\right)+n\left(s^{\prime}\right)}(t) \\
& \left.+(t-1)^{n_{m}} g_{\left\{n\left(S^{\prime}\right)-2 n\left(s^{\prime}\right)-n_{m}\right\}, k-n\left(s^{\prime}\right)-n_{m}}(t)\right] \\
& =g_{\{n(S)\}, k}(t)+(t-1)^{n\left(S^{\prime}\right)} g_{\left\{n(S)-2 n\left(S^{\prime}\right)\right\}, k-n\left(S^{\prime}\right)}(t) \\
& +(t-1)^{n_{m}} g_{\left\{n(S)-2 n_{m}\right\}, k-n_{m}}(t) \\
& +\sum_{s^{\prime}}\left[(t-1)^{n\left(s^{\prime}\right)} g_{\left\{n(S)-2 n\left(s^{\prime}\right)\right\}, k-n\left(s^{\prime}\right)}(t)\right] \\
& +(t-1)^{n\left(S^{\prime}\right)-n\left(s^{\prime}\right)} g_{\left\{n(S)-2\left(n\left(S^{\prime}\right)-n\left(s^{\prime}\right)\right)\right\}, k-\left(n\left(S^{\prime}\right)-n\left(s^{\prime}\right)\right)}(t) \\
& +(t-1)^{n\left(s^{\prime}\right)+n_{m}} g_{\left\{n(S)-2\left(n\left(s^{\prime}\right)+n_{m}\right)\right\}, k-\left(n\left(s^{\prime}\right)+n_{m}\right)}(t) \\
& =g_{\{n(S)\}, k}(t)+\sum_{S}(t-1)^{n(s)} g_{\{n(S)-2 n(s)\}, k-n(s)}(t) \text {. }
\end{aligned}
$$

\section{REFERENCES}

1. D. E. Barton and F. N. David, Runs in a ring, Biometrika 45 (1958), 572-578.

2. F. N. David and D. E. Barton, Combinatorial chance, Hafner, New York, 1962.

3. F. K. Hwang, Blocking probabilities for a class of spiderweb channel graphs, IEEE Trans. Comm. 28 (1980), 115-117.

4. M. A. Stephens, Whitworth runs of a circle, Ann. Inst. Statist. Math. Part A 29 (1977), 287-293.

5. K. Takagi, A generalization of optimal channel graphs in multistage link systems, Japanese Elec. Assoc. SE 77-105 (1978), 39-46. (Japanese)

6. __ A comparison of channel graphs for link system design, Trans. IECE Japan 61 (1978), 538-539.

7. W. A. Whitworth, Choice and chance, Deighton Bell, Cambridge, Mass., 1886.

Bell laboratories, Murray Hill, New Jersey 07904 